\title{
Kernos
}

Revue internationale et pluridisciplinaire de religion grecque antique

13 | 2000

Varia

\section{Marcel DETIENNE, Apollon le couteau à la main. Une approche expérimentale du polythéisme grec}

\section{André Motte}

\section{OpenEdition \\ Journals}

Édition électronique

URL : http://journals.openedition.org/kernos/1314

DOI : 10.4000/kernos.1314

ISSN : 2034-7871

\section{Éditeur}

Centre international d'étude de la religion grecque antique

Édition imprimée

Date de publication : 1 janvier 2000

ISSN : 0776-3824

\section{Référence électronique}

André Motte, « Marcel DETIENNE, Apollon le couteau à la main. Une approche expérimentale du polythéisme grec », Kernos [En ligne], 13 | 2000, mis en ligne le 16 juin 2011, consulté le 21 septembre 2020. URL : http://journals.openedition.org/kernos/1314 ; DOI : https://doi.org/10.4000/kernos.1314 
caces. Les autres cultes de Messénie, sur lesquels les données sont maigres, font l'objet du chapitre 7 (divinités mycéniennes, Aphrodite et Héphaistos, Thétis, Leukothéa, Tyché, les fleuves Achéloos et Pamisos, "les Dieux »), suivi de quelques pages sur les cultes orientaux. La seconde partie du livre, beaucoup plus brève (p. 251-286), traite des héros : héros nationaux (Messéné, Leukippos, Idès, Aristomène), Héraklès, héros guérisseurs et daimones (Tityros).

Au total, l'ouvrage donne une documentation très utile sur les panthéons mycénien et classico-hellénistique. Pour la période qui va de la chute de Pylos à la fondation de Messène, il propose des pistes (caractère ancien des légendes, attestations dans l'apoikia de Messana ou à Naupacte) qui peuvent laisser supposer l'existence de tel ou tel culte. On a là, malgré les incertitudes qui pèsent parfois sur les analyses de détail, un ouvrage particulièrement utile.

\section{Madeleine Jost \\ Madeleine Jost}

Marcel Detienne, Apollon le couteau à la main. Une approche expérimentale du polytbéisme grec, Paris, Gallimard, 1998. 1 vol. $14 \times 22,5 \mathrm{~cm}, 350$ p. (Bibliotbèque des Sciences bumaines). ISBN : 2-07-073371-8.

À défaut de pouvoir encore effrayer, ce titre insolite, qu'illustre une magnifique représentation sur céramique du peintre de Penthésilée, est sans nul doute destiné à surprendre. Mais là n'est pas sa seule raison d'être. L'A. s'en explique dans son «Ouverture " (Apollon, pourquoi pas ?). Il s'agit de redresser une image édifiante et bien ennuyeuse du dieu que, depuis Winckelmann, relayé notamment par W. Otto dans Les Dieux de la Grèce, l'érudition moderne n'a cessé de véhiculer : « décrire Apollon, écrivait le premier, exige le style le plus haut : une élévation au-dessus de tout ce qui est humain ». On savait $M$. Detienne très enclin, au départ de ses ouvrages, à se donner des cibles pour mieux marquer sa différence. Sa critique est ici très mesurée dans le ton, eu égard sans doute à la qualité re connue par ailleurs à cet ouvrage d'Otto dont il a préfacé la traduction française. Il n'empêche que, sous le couvert plus discret des notes, certains philologues sont davantage étrillés; ainsi J. Defradas est-il soupçonné d'avoir, dans son interpré tation de la mort de Néoptolème, transformé "Apollon en Sacré-Cour, au-delà du Dieu moral qui a la peau si dure dans les évocations de la Grèce immortelle, entre académiciens de tout sexe » (p. 315, n. 77)!

L'humour, n'est pas déplacé quand on disserte de dieux au rire inextinguible, et il est rare, reconnaissons-le, de le découvrir ici aussi grinçant. Du moins met-il bien le doigt sur ce qui insupporte particulièrement l'A. : le parti-pris de gommer ou d'épurer, contre l'évidence parfois, ce qui, dans les sources anciennes, viendrait à contredire l'image qu'on s'est faite d'un dieu parfait de la lumière et de la sagesse. Si M. Detienne a choisi pour sa part de mettre en relief telle face noire du Delphien, en le " prenant de biais, par surprise, de nuit ", ce n'est pas, explique-til, pour l'affronter à sa clarté, mais pour rendre compte plus fidèlement, en suivant ses gestes et ses pratiques, de " la texture d'un système polythéiste dont il fait partie » au même titre que les autres Olympiens.

Le sous-titre annonce clairement ce programme méthodologique au sujet duquel l'auteur s'est d'ailleurs expliqué plus longuement dans un récent numéro de Kernos $(10,1997$, p. 57-72). Il s'agit de pratiquer « la micro-analyse d'une coupe prélevée dans ce tissu à dieux multiples ». G. Dumézil est, dans cette perspective, crédité d'un double apport original dont on peut encore aujourd'hui s'inspirer : d'une part, il s'est montré attentif aux groupements récurrents ou circonstanciels faisant apparaître des complémentarités, des antithèses, des hiérarchies; d'autre part, il a souligné le fait qu'en régime polythéiste, un dieu ne peut 
être appréhendé en termes statiques, mais en tenant compte de l'ensemble des positions qu'il occupe et qu'il faut donc commencer par inventorier au lieu de s'arrêter d'emblée aux traits les plus en vue; on se gardera donc de négliger les formes d'association qui apparaissent incidemment et celles que peut aussi suggérer des éléments de la culture ambiante. Cette première forme d'expérimentation se double d'une autre qui consiste à se focaliser sur des éléments concrets, objets, gestes, modes d'action, situations. C'est à la méthode ethnologique d'un Lévi-Strauss qu'il est ici fait référence, mais on pourrait tout aussi bien invoquer derechef sur ce point la démarche dumézilienne. L'avant-propos fait encore état de l'expérience acquise par l'A. dans les enquêtes comparatives qui lui ont appris non pas à généraliser, mais à affiner l'analyse en repérant notamment les petits systèmes différenciés. Dumézil à nouveau n'est pas loin. Aussi bien cet Apollon est-il le fruit d'un cheminement déjà long, jalonné d'une dizaine de publications dont sont rappelés les titres et la genèse. L'ouvrage était effectivement annoncé depuis plusieurs années.

Sept chapitres dessinent le parcours ainsi programmé. Le premier est surtout consacré à une lecture sélective de l'Hymne bomérique à Apollon, dans les deux parties qui le composent et qui sont envisagées dans une perspective unitaire. Il sera souvent question encore de cette ouvre dans la suite car elle est révélatrice d'au moins deux traits qui caractérisent la manière récurrente d'être et d'agir d'Apollon : d'une part, un dieu à l'arc aux allures arrogantes et brutales, qui sait se faire à l'occasion meurtrier et qui, dans les premières recommandations faites à ses prêtres, évoque avec une évidente complaisance le couteau qui servira à égorger d'innombrables victimes, d'autre part, un dieu en marche qui, à l'intérieur des espaces qu'il ouvre et délimite, se fait volontiers bâtisseur de temples et d'autels, bref un dieu épris à la fois de mouvement et de stabilité, celle des seuils monumentaux de pierre. On est ainsi conduit non seulement à Délos et à Delphes, mais aussi à Milet, à Sparte, en Troade, à Cyrène, et aussi dans l'Olympe, où le pas d'Apollon fait verdir les dieux, ainsi qu'au pays des Hyperboréens d'où proviennent deux architectes dont le nom rappelle des épiclèses du dieu : Aguieus et Pagasios.

Comme il le fera souvent à propos d'autres traits, - et ce n'est pas là le moindre mérite de la recherche, - l'A. s'est mué, chaque fois qu'il le fallait, en spécialiste des vocabulaires de la marche et de l'architecture pour montrer combien ils collent aux évocations du dieu voyageur et bâtisseur. C'est son talent de conteur, en revanche, qu'il déploie dans le deuxième chapitre pour évoquer la fête pure ( hagnos) d'Apollon, - une contre-théoxénie en somme, - au cours de laquelle le sang des prétendants impies et ignorants des règles d'hospitalité est répandu en abondance par l'arc vengeur et sans pitié d'Ulysse, un protégé du dieu. L'Apollon de l'Odyssée, pas plus d'ailleurs que celui de l'Iliade, n'est précisément ce qu'on appelle un tendre; la chose n'avait nullement échappé du reste aux exégètes d'Homère. Plus originale est à coup sûr la section suivante qui, dans la foulée de cette effusion de sang qui réjouit le dieu, fait voir un Apollon vorace et friand d'hécatombes, promenant, dans la Pyla de Chypre, sa sensualité en compagnie de ses prêtres-bouchers : il y préside à la fête des viandes grésillantes et des chairs éclatées, on l'appelle en personne Mageirios, le boucher-sacrificateur, le technicien de l'égorgement et de la découpe. Mais c'est en bien d'autres lieux encore qu'on découvre ce dieu de l'arrière-cuisine, l'occasion pour l'A. de disserter aussi, à l'aide d'un mythe que rapporte Porphyre (De abstinentia), de l' " envie irrésistible de goûter de la viande », des grésillements du feu et de l'empyromantie, des fumets et des vapeurs de graisse, des gargotiers de Delphes et des restes du Dionysos mis à mort dont l'Apollon delphique prend le plus grand 
soin. C'est peut-être dans cette espèce de traité de la gourmandise apollinienne que l'on a le plus de surprises. Mais serait-il l'apanage du seul dieu de Pytho?

L'Apollon des bouchers n'est pas loin, nous dit-on, du dieu des meurtriers, proche lui-même de ce marcheur violent venu s'établir au pied du Parnasse. Mais il s'agit de faire voir à présent, dans les chapitres IV et V, Apollon en prince de la colonisation, archégète vénéré comme tel dans pas moins de quinze cités connues et guide attitré (bègemôn), grand exégète encore qui trace pareillement les chemins de la parole oraculaire, celui que l'on consulte avant toute fondation de colonie. La dimension politique de cette œuvre est rendue notamment manifeste par le fait que c'est à lui ainsi qu'à Poseidon et à Hestia, symbole du foyer public, que s'adresse le premier sacrifice exécuté par l'œciste à l'aide de la marmite de feu emmenée du prytanée de la cité-mère. Ce n'est pas au profit des Olympiens qu'Apollon fonde des cités, mais il fait carrière au milieu des hommes, se tenant en avant des cités (Proasterios) pour mieux les protéger (Apotropaios). L'A. s'attache ensuite à montrer comment le politique se cache derrière le civilisateur, chose dont les hellénistes se sont depuis longtemps avisés, note-t-il, mais souvent sans chercher à se justifier.

De belles pages sont encore consacrées aux Chemins de la parole, dans les pas de la déesse Thémis, un chapitre (VI) qu'on se réjouit de pouvoir confronter à la monographie récente de J. Rudhardt, dont il est également rendu compte ici. Une attention particulière est accordée aux différents sens, souvent concrets, des mots apparentés à celui de cette déesse nourrice d'Apollon, l'examen de cette configuration sémantique permettant d'éclairer la nature de la parole oraculaire du dieu et ses modes de cheminement.

Pendant un long temps donc, Apollon a rengainé son couteau, mais revoici cet instrument dans l'ultime chapitre (L'architecte du pur et de l'impur), apportant au titre de l'ouvrage une justification nouvelle au prix de quelques investigations supplémentaires et aussi d'une métaphore suggestive. Le couteau à la main, c'est celui certes qui égorge, l'homme ou l'animal, mais c'est aussi celui qui défriche et qui taille, qui ouvre les chemins, trace le temenos, découpe les autels

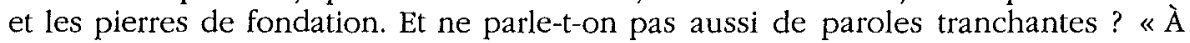
la croisée des chemins du couteau et de la parole, Apollon règne sur le pur et l'impur... Son art extrême n'est pas de purifier mais de faire du pur avec de l'impur, montrant hardiment comment, depuis le plus informe, se donner un tracé sans mémoire, créer le pur recommencement d'une fondation qui veut se croire durable. »

Il n'est pas sûr que l'on rende service à un tel ouvrage en produisant un résumé qui ambitionne d'en rendre compte en une page. On n'a fait qu'extraire, en effet, avec tout l'arbitraire que cela peut souvent comporter, quelques idées, et quelques faits sur lesquelles elles s'appuient, d'une documentation foisonnante dont les divers éléments, parfois très disparates, mais toujours savamment combi nés et répartis à la façon d'un architecte - c'était bien le moins! -, ont chacun une place à faire valoir dans la démonstration. M. Detienne, comme bien souvent, a ici beaucoup lu et scruté, produisant quantité de données peu connues ou pas connues du tout, et apportant surtout de nouvelles façons de regarder. On ne laisse pas d'être impressionné, d'autre part, par l'appareil d'érudition qui s'étale sur près de cent pages et comprend plus de quatorze cents notes, mais, pour le dire en passant, on est aussi gêné par le constant chassé croisé qu'impose le report de celles-ci en fin de volume (si cette règle est imposée, mieux vaudrait changer de collection ou d'éditeur !).

La démonstration est brillante et le résultat est convaincant si l'objectif était de rééquilibrer, en mettant en évidence ses facettes obscures et moins rassurantes, 
l'image traditionnelle qu'on s'est faite jusqu'ici d'Apollon. Il n'est pas sûr du tout qu'à la longue ce dieu marcheur et égorgeur soit de nature à faire bâiller moins de classes que ne le fait aujourd'hui, paraît-il, le dieu de la sagesse dès qu'est prononcé son nom. Mais ne préjugeons pas ici de l'avenir ! Il n'est pas sûr non plus que la méthode annoncée ait tenu toutes ses promesses. C'est le cas notamment du dessein de définir Apollon dans la texture d'un système polythéiste. Il y a bien çà et là, d'utiles remarques à propos des rapports entre Apollon et certains de ses congénères, comme Poseidon et Hestia, mais il n'apparaît pas que toutes les possibilités qu'offraient les textes utilisés, à commencer par l'Hymne boméri$q u e$, aient été de ce point de vue exploitées. Tout compte fait, on n'est pas, de ce point de vue, si éloigné du genre monographique que critique volontiers l'A. Et que dire ici de son idée d'expérimentation? Il en convient : nous n'avons pas le pouvoir de faire se répéter les phénomènes observés comme on le fait dans un laboratoire, ni non plus de modifier les conditions de l'expérience. Dès lors, le terme de comparaison qui conviendrait le mieux en l'occurrence ne serait-il pas le travail méthodique qu'on opère sur un champ de fouilles lorsqu'on s'efforce, par essais et erreurs, de reconstituer à partir des éléments mis au jour tantôt des objets, tantôt des ensembles plus importants et plus complexes comme sont les sanctuaires? Il y a bien un peu de cela dans la méthode préconisée qui consiste à observer comment réagissent les dieux par rapport à tels objets concrets, tels gestes, telles situations, pour y découvrir, sous-jacente, une sorte de logique. $\grave{A}$ noter toutefois que l'archéologue, pour sa part, a commencé par noter soigneusement les strates d'où proviennent les objets récoltés; il n'aurait pas idée de réunir d'emblée, en vue de reconstituer un vase, des fragments découverts à des niveaux fort éloignés. Se pourrait-il que, pour faire réagir des dieux à des objets, à des situations, on soit autorisé à les considérer dans un éternel présent, comme si, avec le temps, rien ne changeait autour d'eux et comme si eux-mêmes ne faisaient pas l'objet d'appréhensions différentes ? Il y aurait là, parmi d'autres, une question qu'on aimerait poser à $M$. Detienne.

Peut-être ne tardera-t-on pas à l'obtenir car cet Apollon ne sera pas le dernier ouvrage du genre, l'A. méditant à présent, ainsi qu'il l'annonce en conclusion, d'expérimenter dans l'épais dossier de la configuration, ô combien complexe, que forment ensemble les demi-frères Apollon et Dionysos. Nul n'y est sans doute mieux préparé que lui; on souhaitera seulement que l'investigation n'ait pas l'ampleur du Zeus de Cook!

André Motte

(Université de Liège)

Giovanni Casadio, Il vino dell'anima. Storia del culto di Dioniso a Corinto, Sicione, Trezene, Roma, Editrice "il Calamo", 1999. 1 vol. $17 \times 24 \mathrm{~cm}, 231 \mathrm{p}$. (Biblioteca di Storia delle Religioni, 1). ISBN : 88-86148-54-2.

Après sa Storia del culto di Dioniso in Argolide, l'A. a réuni dans cet ouvrage plusieurs contributions ayant pour thème général Dionysos. Dans le long prologue intitulé «Dioniso e il vino », l'A. tente de montrer que les liens qui unissent Dionysos au vin remontent à la plus haute antiquité et qu'il apparaîtrait déjà comme dieu du vin dans les tablettes en Lin. B. Contrairement à l'opinion défendue par U. von Wilamowitz-Møellendorff et d'autres (O. Kern, M. Müller, M.P. Nilsson, H. Jeanmaire) pour qui Dionysos serait primitivement un dieu de la végétation se spécialisant ultérieurement dans le vin, celui-ci constituerait, dès le milieu du $2^{\mathrm{e}}$ millénaire, une image synecdotique du tempérament profondément ambivalent de Dionysos. L'A. se fonde sur la tablette en linéaire B PK Xa 1419 où cohabitent autour du vin (wo-no) un mot qui en dérive (wo-no-wa-ti-si) men- 